\title{
DEGRADAÇÃO DE BTXS VIA PROCESSOS OXIDATIVOS AVANÇADOS
}

Elaine Regina Lopes Tiburtius e Patricio Peralta-Zamora*

Departamento de Química, Universidade Federal do Paraná, CP 19081, 81531-990 Curitiba - PR

Alexandre Emmel

Centro Integrado de Tecnologia e Educação Profissional, 81310-010 Curitiba - PR

Elenise Sauer Leal

Centro Federal de Educação Tecnológica, CP 20, 84016-210 Ponta Grossa - PR

Recebido em 26/2/04; aceito em 30/7/04; publicado na web em 12/11/04

\begin{abstract}
DEGRADATION OF BTXs BY ADVANCED OXIDATIVE PROCESSES. In this study the efficiency of advanced oxidative processes (AOPs) were investigated toward the degradation of aqueous solutions containing benzene, toluene and xylenes (BTX). The results indicated that BTX can be effectively oxidized by the UV-A-assisted photo-Fenton process. The treatment permits almost total degradation of BTX and removal of more than $80 \%$ of the phenolyc intermediates at reaction times of about 30 min. Preliminary investigations using solar light suggest a good potentiality of the process for the treatment of large volumes of aqueous samples containing these polluting species.
\end{abstract}

Keywords: BTXs; advanced oxidation processes; remediation.

\section{INTRODUÇÃO}

A contaminação de solos e águas subterrâneas por compostos orgânicos voláteis tem sido destaque nas últimas décadas, principalmente em função da frequiência com que episódios de contaminação são verificados e da gravidade com que o meio ambiente é afetado. Embora os grandes vazamentos acidentais de petróleo sejam preocupantes e ocupem grande espaço na mídia, estima-se que a principal fonte de contaminação por estas espécies seja devida a pequenos e contínuos vazamentos de combustível em postos de distribuição ${ }^{1}$, favorecidos pelo envelhecimento dos taques de estocagem.

No Brasil existem cerca de 27.000 postos de combustíveis, muitos dos quais operando em condições de risco. Em razão deste fato, o potencial poluente desta atividade mostra-se bastante elevado, o que faz com que a contaminação de aquíferos seja apenas uma questão de tempo ${ }^{2}$. A preocupação relacionada com o potencial de contaminação de águas subterrâneas vem crescendo em diversas cidades, principalmente São Paulo, Curitiba e Joinville. Estudos realizados pela prefeitura de Joinville em 65 postos da cidade revelaram que somente um dos postos examinados não apresentava problemas de vazamento que redundasse em contaminação do lençol freático ${ }^{3}$.

O potencial poluente da gasolina está diretamente relacionado com os hidrocarbonetos aromáticos de maior solubilidade em água, isto é, benzeno, tolueno e xilenos (BTXs). Dentre estes, o benzeno mostrase especialmente tóxico, podendo provocar câncer quando em condições de exposição crônica, principalmente devido à absorção por via respiratória ${ }^{4}$. Além disso, a gasolina comercializada em nosso país é aditivada com cerca de 20 a $25 \%$ de etanol, fator que aumenta consideravelmente a solubilidade destes poluentes e, conseqüentemente, a sua migração em corpos d'água ${ }^{5}$. Adicionalmente, estudos recentes têm demonstrado que a biodegradação do etanol consome rapidamente todos os aceptores de elétrons disponíveis no meio, o que faz com que a biodegradação dos BTXs seja drasticamente afetada ${ }^{6}$. Em função destes antecedentes, a busca de novas alternativas para a remediação de águas contaminadas mostra-se absolutamente essencial.

*e-mail: zamora@quimica.ufpr.br
Nos últimos anos, os processos oxidativos avançados (POAs) têm aparecido como uma excelente alternativa para o tratamento de resíduos, principalmente em razão da sua elevada eficiência de degradação frente a substratos resistentes. O processo, fundamentado na geração do radical hidroxila $\left(\mathrm{HO}^{*}\right)$ fortemente oxidante, permite a rápida e indiscriminada degradação de uma grande variedade de compostos orgânicos, muitas vezes permitindo a sua completa mineralização ${ }^{7-9}$.

Dentre as várias alternativas existentes para a produção de radical hidroxila pode ser dado destaque à fotocatálise heterogênea, principalmente na presença de $\mathrm{TiO}_{2}{ }^{10,11}$, fotólise assistida por peróxido de hidrogênio ${ }^{12,13}$ e sistemas Fenton ${ }^{14-16}$, processos estes bastante explorados para a degradação de inúmeros poluentes orgânicos de relevância.

O objetivo deste trabalho consiste em avaliar a potencialidade dos processos oxidativos avançados $\left(\mathrm{TiO}_{2} / \mathrm{UV}, \mathrm{UV} / \mathrm{H}_{2} \mathrm{O}_{2}\right.$, Fenton e foto-Fenton), em relação à degradação de soluções aquosas contendo benzeno, tolueno e xilenos (BTXs).

\section{PARTE EXPERIMENTAL}

\section{Materiais}

Benzeno (Biotec ${ }^{17}$ ), tolueno $\left(\right.$ Merck $\left.^{18}\right)$ e xileno $\left(\right.$ Merse ${ }^{19}$ ) foram utilizados sem nenhum processo de purificação prévio, como soluções aquosas contendo $20 \mathrm{mg} \mathrm{L}^{-1}$. Peróxido de hidrogênio foi utilizado em solução aquosa a $10 \%(\mathrm{~m} / \mathrm{v})$, preparada a partir de uma solução estoque a $50 \%$, gentilmente fornecida pela Peróxidos do Brasil Ltda ${ }^{20}$. Dióxido de titânio P-25 (Degussa ${ }^{21}$ ), $\mathrm{O}_{2}$ de grau comercial (White Martins ${ }^{22}$ ) e sulfato ferroso $\left(\mathrm{FeSO}_{4} \cdot 7 \mathrm{H}_{2} \mathrm{O}\right.$, Isofar ${ }^{23}$ ) foram utilizados como recebidos.

\section{Tratamento fotoquímico}

O tratamento de soluções de benzeno e BTXs foi realizado em um reator fotoquímico de bancada de $300 \mathrm{~mL}$ de capacidade (Figura 1), equipado com resfriamento a água, agitação magnética e sistema de oxigenação (vazão de aproximadamente $45 \mathrm{~mL} \mathrm{~min}{ }^{-1}$ ). A radia- 
ção foi proporcionada por lâmpada a vapor de mercúrio $(125 \mathrm{~W}$, Philips ${ }^{24}$ ), inserida na solução por meio de um bulbo de quartzo (para radiação UV) ou de vidro (para radiação UV-A).

Nos estudos envolvendo fotocatálise heterogênea, o fotocatalisador $\left(\mathrm{TiO}_{2}\right)$ foi utilizado na forma de suspensão $\left(50 \mathrm{mg} \mathrm{L}^{-1}\right)$.

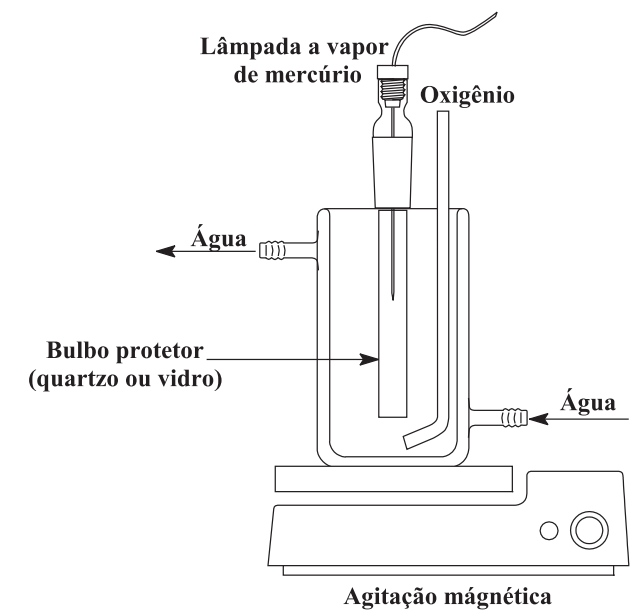

Figura 1. Reator fotoquímico

\section{Controle analítico}

As concentrações de benzeno, tolueno e xileno foram determinadas por cromatografia gasosa (sistema "headspace"), utilizando cromatógrafo Varian (modelo CP3800), equipado com detector de ionização de chama e coluna DB624 (6\% cianopropilfenil, 94\% dimetilsiloxano). Para a aplicação do "headspace" foi utilizado tempo de incubação de $30 \mathrm{~min}$, à temperatura de $70^{\circ} \mathrm{C}$. Curvas de calibração individuais foram preparadas a partir de solução estoque de $30 \mathrm{mg} \mathrm{L}^{-1}$ de benzeno, tolueno e xileno em metanol.

As determinações de peróxido de hidrogênio residual, fenóis totais e $\mathrm{Fe}^{2+} / \mathrm{Fe}^{3+}$ foram realizadas espectrofotometricamente, utilizando-se metodologias fundamentadas na reação com vanadato de amônio $^{25}$, reagente de Folin-Ciocalteau (método padrão APHA $^{26}$ ) e orto-fenantrolina ${ }^{27}$, respectivamente. As medidas foram realizadas em espectrofotômetro S-1150 SINCO, utilizando-se cubetas de quartzo de $1 \mathrm{~cm}$.

\section{RESULTADOS E DISCUSSÃO}

\section{Degradação fotoquímica de benzeno}

Dentre as três espécies químicas em estudo, o benzeno costuma ser considerado o mais resistente frente aos processos de remediação convencionais $^{28}$. Por este motivo foi utilizado como substrato modelo em todos os estudos preliminares de otimização e caracterização dos processos oxidativos propostos neste trabalho. Desta forma, o efeito de cada parâmetro experimental de importância (ex. pH, concentração de reagentes, concentração de substratos, etc.) foi investigado através de planejamento fatorial dos experimentos, utilizandose a degradação de benzeno como resposta analítica. Seguidamente, todos os processos foram aplicados em condições previamente adequadas, obtendo-se os resultados apresentados a seguir.

O processo de degradação fotoquímica de benzeno inicia-se pela adição do radical hidroxila à dupla ligação do anel aromático, gerando intermediários de natureza fenólica ${ }^{14,29}$. Assim, o monitoramento destas espécies durante a degradação pode trazer importantes informações, principalmente quando se deseja avaliar diferenças mecanísticas entre processos de oxidação. Adicionalmente, a determina- ção de fenóis totais mostra-se bastante conveniente para o monitoramento de processos deste tipo, processos estes, em que tanto a cromatografia gasosa, como a espectroscopia UV-VIS, mostram-se inadequadas, em razão do caráter polar dos intermediários e da interferência de espécies que absorvem fortemente na mesma região espectral dos BTXs, respectivamente.

Os resultados obtidos na determinação de fenóis totais refletem, de forma qualitativa, o avanço do processo de degradação. É evidente na Figura 2 que o processo foto-Fenton é o mais efetivo do ponto de vista cinético, permitindo um pico de geração de formas fenólicas em tempos da ordem de $5 \mathrm{~min}$, espécies estas que são completamente degradadas em tempos não superiores a $15 \mathrm{~min}$. No outro extremo, o processo Fenton mostra-se bastante ineficiente nos primeiros minutos monitorados, provocando um pico de formas fenólicas em tempos de reação da ordem de $60 \mathrm{~min}$. Os processos restantes mostram uma eficiência intermediária, caracterizada por uma lenta formação de intermediários fenólicos, que degradam, também, lentamente.

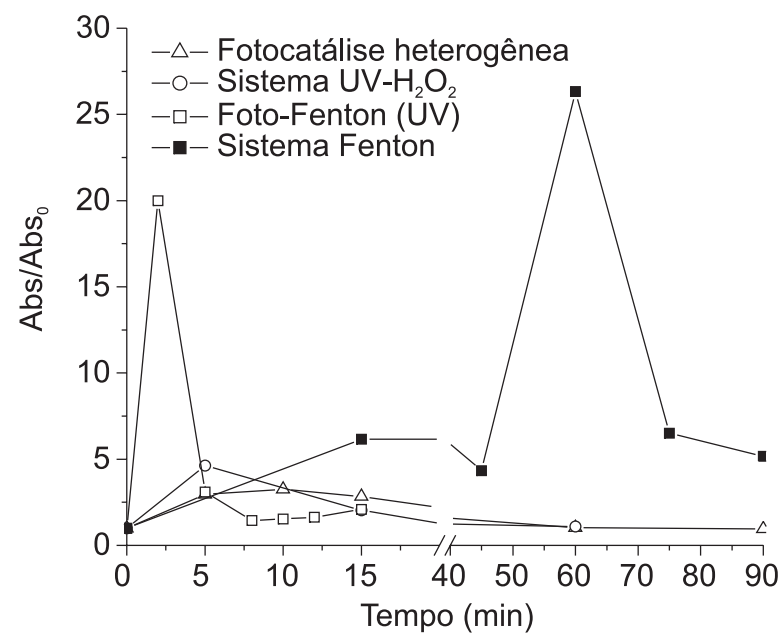

Figura 2. Variação da concentração $\left(A b s / A b s_{0}\right)$ de fenóis totais durante a degradação de benzeno $\left(20 \mathrm{mg} \mathrm{L}^{-1} ; 250 \mathrm{~mL}\right)$; $\mathrm{TiO}_{2} / \mathrm{UV}\left(\mathrm{TiO}_{2}: 50 \mathrm{mg} \mathrm{L}^{-1}\right.$; $\mathrm{pH}$ 6); $\mathrm{UV}_{\mathrm{H}} \mathrm{O}_{2}\left(\mathrm{H}_{2} \mathrm{O}_{2}: 100 \mathrm{mg} \mathrm{L}^{-1} ; \mathrm{pH} \mathrm{6}\right) ;$ Fenton e foto-Fenton $\left(\mathrm{Fe}^{2+}=10 \mathrm{mg}\right.$ $\left.\mathrm{L}^{-1} ; \mathrm{H}_{2} \mathrm{O}_{2}=100 \mathrm{mg} \mathrm{L}^{-1} ; \mathrm{pH} \mathrm{3}\right)$

Além de mostrar uma eficiência de degradação bastante acentuada, que permite redução significativa no tempo de tratamento, o sistema foto-Fenton oferece algumas vantagens adicionais, associadas, por exemplo, à simplicidade operacional que a sua natureza homogênea permite e à possibilidade de ser operado com radiação UV-A. Trata-se de fatores decisivos no momento em que há necessidade de ampliação de escala.

Visando verificar a eficiência do sistema foto-Fenton sob diferentes condições de irradiação, foram conduzidos estudos de degradação de benzeno, obtendo-se os resultados apresentados na Figura 3. Estes resultados demonstram que a radiação ultravioleta proporciona uma cinética de degradação mais favorável, principalmente nos primeiros minutos de reação. Trata-se de um resultado esperado, uma vez que, além de catalisar a fotorredução de íons $\mathrm{Fe}^{3+}$ (ver Equação 1), a radiação ultravioleta permite a decomposição do peróxido de hidrogênio (ver Equação 2), o que implica na formação de maior quantidade de radical hidroxila ${ }^{30}$.

$\mathrm{Fe}^{3+}+\mathrm{H}_{2} \mathrm{O}+\mathrm{h} v \rightarrow \mathrm{Fe}^{2+}+\mathrm{H}^{+}+\mathrm{HO}^{\cdot}$

$\mathrm{H}_{2} \mathrm{O}_{2}+\mathrm{hv} \rightarrow 2 \mathrm{HO}^{\cdot}$

Entretanto, os resultados também sugerem que o sistema fotoFenton operado com radiação no ultravioleta próximo (UV-A: 300- 


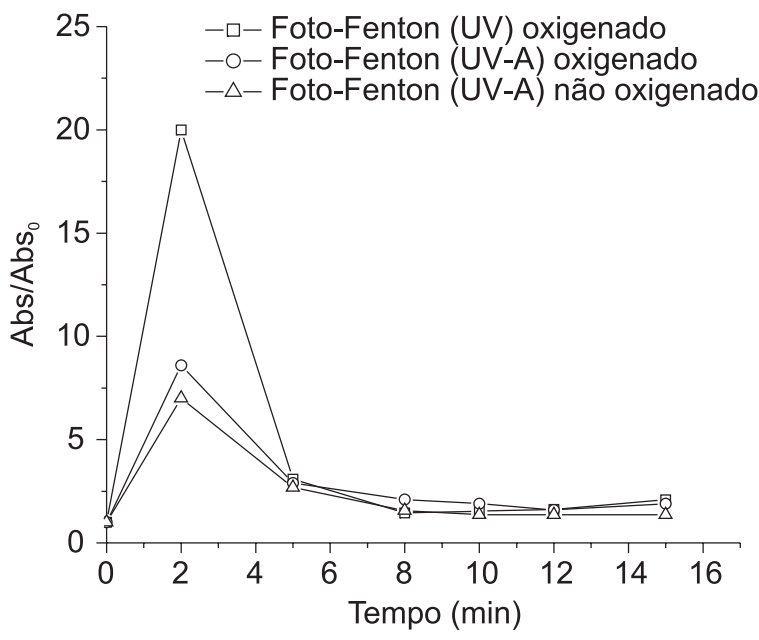

Figura 3. Variação da concentração de fenóis totais (Abs/Abs $)_{0}$ durante a degradação de benzeno pelo sistema foto-Fenton UV e UV-A (Benzeno= 20 $\left.m g L^{-1}, 250 \mathrm{~mL} ; \mathrm{Fe}^{2+}=10 \mathrm{mg} \mathrm{L}^{-1} ; \mathrm{H}_{2} \mathrm{O}_{2}=100 \mathrm{mg} \mathrm{L}^{-1} ; \mathrm{pH} 3\right)$

400 nm), com cinética inicial menos favorável, permite a obtenção de resultados comparáveis, para tempos de reação algo maiores (10 min). Adicionalmente, estudos paralelos demonstraram a inexistência de diferenças significativas quando o sistema foto-Fenton (UV-A) é operado com ou sem oxigenação (Figura 3). Estes dois resultados se revestem de grande importância, uma vez que permitem reduzir de maneira significativa o custo operacional do sistema.

Apesar da molécula de benzeno ser bastante resistente aos tratamentos convencionais, principalmente em razão da estabilidade que o efeito de ressonância lhe confere, a elevada eficiência de degradação do sistema foto-Fenton foi demonstrada neste trabalho, mesmo nas condições brandas representadas pelo sistema não-oxigenado e assistido por radiação UV-A. Em razão destes resultados promissores, a degradação de BTXs foi estudada em sistemas foto-Fenton, operados com radiação UV-A e na ausência de oxigenação.

\section{Degradação fotoquímica de BTXs}

Acompanhando-se o processo de degradação por cromatografia gasosa (Figura 4), foi possível observar que a concentração de BTXs foi reduzida até valores menores que o limite da determinação ( $\left.5 \mu \mathrm{g} \mathrm{L}^{-1}\right)$, em apenas $5 \mathrm{~min}$ de tratamento. Além disso, a técnica cromatográfica ("headspace") não revelou o surgimento de intermediários voláteis, o que implica que o processo fotoquímico induz uma rápida modificação estrutural dos compostos de partida, com formação de espécies de maior polaridade. Embora intermediários de caráter fenólico sejam esperados, o resultado antes comentado tem grande importância, uma vez que os critérios legais de potabilidade estabelecem em $5 \mu \mathrm{g} \mathrm{L}^{-1}$ a concentração máxima permitida para o benzeno ${ }^{31}$.

O acompanhamento da concentração de espécies fenólicas demonstra a geração de intermediários desta natureza, que são degradados, em sua maioria, em tempos de reação de 30 min (Figura 4). Adicionalmente, o acompanhamento da concentração de peróxido de hidrogênio residual atesta um consumo superior a $95 \%$, em igual tempo de reação, fato que garante não apenas a eliminação dos substratos de interesse, mas também de qualquer resíduo potencialmente perigoso.

A eficiência elevada do sistema nos primeiros minutos de reação pode ser atribuída a uma elevada eficiência inicial do sistema Fenton, o qual, além de permitir a formação de um equivalente de radical hidroxila, leva à geração de íons férricos. Nas condições em que o processo é aplicado (solução aquosa e pH 3), os íons férricos $\left(\mathrm{Fe}^{3+}\right)$

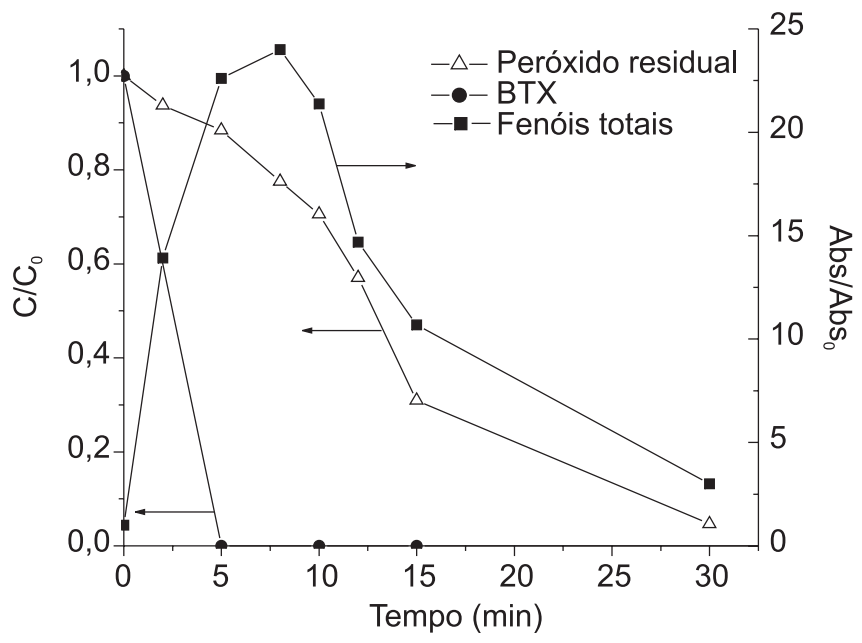

Figura 4 . Variação da concentração de BTXs, fenóis totais e peróxido residual $\left(C / C_{0}\right)$ durante a degradação dos BTXs (BTXs $=20 \mathrm{mg} \mathrm{L}^{-1}, 250 \mathrm{~mL}$; $\left.\mathrm{Fe}^{2+}=10 \mathrm{mg} \mathrm{L}^{-1} ; \mathrm{H}_{2} \mathrm{O}_{2}=100 \mathrm{mg} \mathrm{L}^{-1} ; \mathrm{pH} \mathrm{3}\right)$

encontram-se predominantemente na forma $\mathrm{Fe}(\mathrm{OH})^{2+30}$, espécie que absorve notavelmente radiação de comprimento de onda maior que $300 \mathrm{~nm}^{32}$. A absorção de radiação leva à fotorredução dos íons férricos, com formação de íons ferrosos e geração de mais um equivalente de radical hidroxila (Equação 3). Este processo fecha um ciclo catalítico que maximiza a eficiência do processo degradativo. Após 15 min de reação a conversão ferroso-férrico desvia do padrão dos primeiros minutos, provavelmente em razão da baixa concentração residual do peróxido de hidrogênio.

$\mathrm{Fe}(\mathrm{OH})^{2+}+\mathrm{h} v \rightarrow \mathrm{Fe}^{2+}+\mathrm{HO}^{\bullet}$

A elevada eficiência observada nos sistemas operados com radiação UV-A sugere uma boa potencialidade dos processos foto-Fenton assistidos por radiação solar. Para verificação desta premissa alguns testes preliminares foram realizados de maneira bastante simples, utilizando-se béquer de vidro Pyrex exposto diretamente à radiação

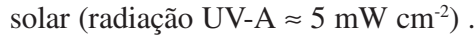

Os resultados (Figura 5) mostram uma rápida degradação dos BTXs, o que permite alcançar concentrações residuais de $20 \mu \mathrm{g} \mathrm{L}^{-1}$

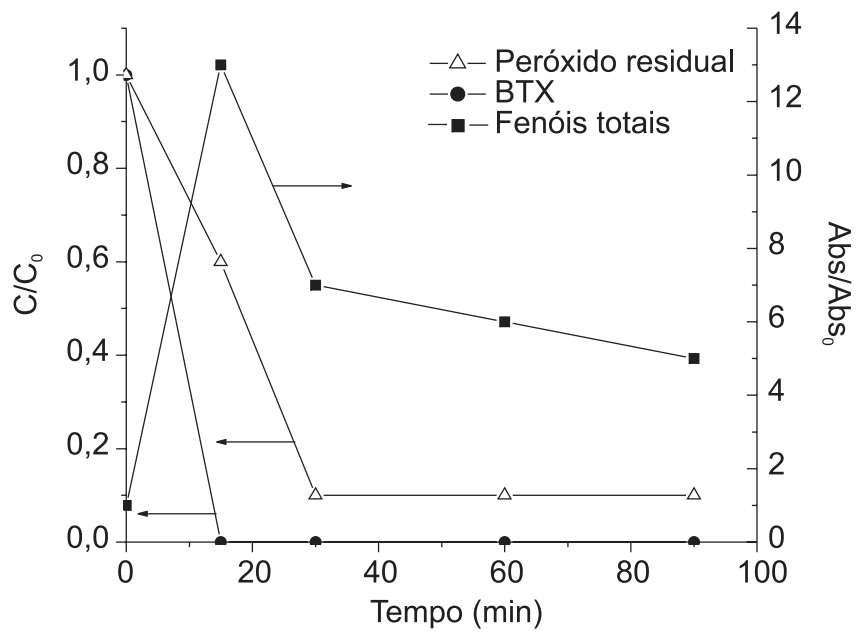

Figura 5. Variação da concentração de BTXs, fenóis totais e peróxido residual $\left(C / C_{0}\right)$ durante a degradação dos BTXs por processo assistido por radiação solar $\left(B T X s=20 \mathrm{mg} \mathrm{L}^{-1}, 250 \mathrm{~mL} ; \mathrm{Fe}^{2+}=10 \mathrm{mg} \mathrm{L}^{-1} ; \mathrm{H}_{2} \mathrm{O}_{2}=100 \mathrm{mg}\right.$ $\left.L^{-1} ; p H 3\right)$ 
(benzeno), $7 \mu \mathrm{L} \mathrm{L}^{-1}$ (tolueno) e $8 \mu \mathrm{g} \mathrm{L}^{-1}$ (xileno), a partir de tempos de reação de 15 min. A evolução de formas fenólicas acompanha o padrão usual, com uma rápida formação nos primeiros minutos de tratamento, seguida de degradação mais lenta, que permite remover aproximadamente $60 \%$ das formas fenólicas geradas inicialmente. A persistência de $40 \%$ destas formas fenólicas, mesmo após 90 min de tratamento, evidencia a menor eficiência das reações assistidas por radiação solar. Entretanto, é importante salientar que o consumo de peróxido de hidrogênio se dá de maneira muito rápida, o que, provavelmente, também contribui com a diminuição da eficiência de degradação.

Finalmente, cabe destacar que a eficiência deste sistema pode ser significativamente melhorada, melhorando-se a geometria do reator (aumento da área irradiada), a eficiência do processo de irradiação (uso de coletores solares) e utilizando-se múltipla adição de peróxido de hidrogênio.

\section{CONCLUSÕES}

Dentre os processos oxidativos avançados investigados neste trabalho, o sistema foto-Fenton assistido por radiação UV-A merece especial destaque. A sua elevada eficiência de degradação permite completa degradação dos substratos de partida (benzeno, tolueno e xileno) e dos intermediários fenólicos, em tempos de reação da ordem de 30 min. Por sua vez, os sistemas assistidos por radiação solar mostram-se bastante promissores, precisando, entretanto, de estudos mais detalhados, que permitam melhorar sua eficiência fotônica.

Finalmente, características como simplicidade operacional, permitida pela sua natureza homogênea, e economia, representada pelo baixo consumo de reagentes, fazem com que o sistema foto-fenton assistido por radiação visível represente uma boa alternativa para a remediação de resíduos contendo espécies químicas resistentes, como as aqui estudadas.

\section{REFERÊNCIAS}

1. Watts, R. J.; Haller, D. R.; Jones, A. P.; Teel, A. L.; J. Hazard. Mater. 2000, 76,73 .

2. Corseuil, H. X.; Marins, M. D. M.; Revista Engenharia Ambiental e Sanitária 1997, 2, 50.
3. Penner, G. C.; Dissertação de Mestrado, Universidade Federal de São Carlos, Brasil, 2000.

4. Römmelt, H.; Pfaller, A.; Fruhman, G.; Nowak, D.; Sci. Total Environ. 1999, 241, 197.

5. Corseuil, H. X.; Hunt, C. S.; Santos, R. C. F.; Alvarez, P. J. J.; Water Res. 1998, 32, 2065.

6. Kulkamp, M. S.; Kaipper, B. I. A.; Corseuil, H. X.; Artigos do $22^{\circ}$ Congresso Brasileiro de Engenharia Sanitária e Ambiental, Joinville, Brasil, 2003.

7. Feng, J.; Hu, X.; Yue, P. L.; Zhu, H. Y.; Lu, G. Q.; Water Res. 2003, 37, 3776.

8. Joseph, J. M.; Varghese, R.; Aravindakumar, C. T.; J. Photochem. Photobiol., A 2001, 146, 67.

9. Safarzadeh-Amiri, A.; Bolton, J. R.; Cater, S. R.; Water Res. 1997, 31, 787.

10. Nogueira, R. F. P.; Jardim, W. F.; Quim. Nova 1998, 21, 69.

11. Pirkanniemi, K.; Sillanpää, M.; Chemosphere 2002, 48, 1047.

12. Juang, L-C.; Tseng, D-H.; Yang, S. C.; Water Sci. Technol. 1997, 36, 357.

13. Chang, P. B. L.; Young, T. M.; Water Res. 2000, 34, 2233.

14. Neyens, E.; Baeyens, J.; J. Hazard. Mater. 2003, 98, 33.

15. De Laat, J.; Gallard, H.; Ancelin, S.; Legube, B.; Chemosphere 1999, 39, 2693.

16. Feitz, A. J.; Guan, J.; Chattopadhyay, G.; Waite, T. D.; Chemosphere 2002, $48,401$.

17. http//www.biotec.com, acessada em Abril 2004.

18. http//www.merck.com.br/quimica, acessada em Abril 2004.

19. http//www.merse.com.br/principal.html, acessada em Abril 2004.

20. http//www.peroxides.com.br, acessada em Abril 2004.

21. http//www.degussa.com.br, acessada em Abril 2004.

22. http//www.whitemartins.com.br/site/home/index.jsp, acessada em Abril 2004.

23. http//www.isofar.com.br/meio.htm, acessada em Abril 2004.

24. http//www.philips.com.br, acessada em Abril 2004.

25. Oliveira, M. C.; Nogueira, R. F. P.; Gomes Neto, J. A.; Jardim, W. F.; Rohwedder, J. J. R.; Quim. Nova 2001, 24, 188.

26. APHA; Standart Methods for Examination of and Wasterwater, $18^{\text {th }}$ ed.; Ed. Amer. Publ. Health Ass no5550p, 5.41-5.42, 1992.

27. Harris, D. C.; Análise Química Quantitativa, $5^{\mathrm{a}}$ ed., LTC: Rio de Janeiro, 2001.

28. Phelps, D. C.; Kazumi, J.; Young, Y. L.; FEMS Microbiol. Lett. 1996, 145, 433.

29. Fujishima, K.; Fukuoka, A.; Yamagishi, A.; Inagaki, S.; Fukushima, Y.; Ichikawa, M.; J. Mol. Catal. A: Chem. 2001, 166, 211.

30. Rodriguez, M.; Timokhin, V.; Michl, F.; Contreras, S.; Gimenez, J.; Esplugas, S.; Catal. Today 2002, 76, 291.

31. http//www.funasa.gov.br/legis/pdfs/portarias_m/pm1469_2000.pdf, acessada em Julho 2003.

32. Feng, W.; Nansheng, D.; Chemosphere 2000, 41, 1137. 